\title{
THE TEMPERATURES OF WHITE DWARFS IN DWARF NOVAE
}

\author{
B. J. M. HASSALL ${ }^{1}$, C. LA DOUS ${ }^{2}$ \\ 1. Centre for Astrophysics, University of Central Lancashire, \\ Preston, PR1 2HE, UK \\ 2. ESA/IUE Observatory, Villafranca del Castillo, Apartado \\ 50727, 28080 Madrid, Spain
}

\begin{abstract}
We attempt to measure the temperature of all the quiescent dwarf novae that have been observed with IUE, by fitting the observed flux distribution of white dwarfs (WDs) with known temperatures. This approach gives results similar to those using much more sophisticated modelling, but allows us to investigate the relationship with system parameters and subclasses.
\end{abstract}

\section{Introduction}

In recent years, there has been an upsurge in interest in measuring the temperature of the white dwarf in dwarf novae as it is fundamental in understanding various aspects of the WD's binary environment and the evolutionary status of CVs. A large number of dwarf novae and other CVs have been observed with IUE, allowing us to determine the temperatures of their white dwarfs. While the results for any one system are not as reliable as the detailed WD atmosphere models applied to HST data, their number allows a statistical approach that will not be possible with HST results for some years to come.

\section{The method}

In quiescence, the flux in the SWP range shortward of about $1600 \AA$ is dominated by emission from the white dwarf (la Dous 1996). In our simple approach, we fit WD spectra to this region of the spectrum, using both the Lyman alpha profile and the continuum shape. Considering that we have not used detailed modelling, it is remarkable what good agreement 
is achieved with the more sophisticated techniques. For example, for OY Car, we find $T_{\mathrm{WD}}=15200 \mathrm{~K}$ while Horne et al. (1994) find the WD is best fitted by a $16500 \mathrm{~K}$ WD with solar metallicity shrouded by a curtain of disk material at $10000 \mathrm{~K}$. From this we deduce that we probably systematically underestimate $T_{\mathrm{WD}}$. Although this difference is not negligible, it is not sufficiently large to invalidate the overall trends seen in our study.

\section{Discussion}

The complete list of the temperatures of those WDs best fitting the dwarf novae spectra can be found in la Dous (1996). When arranged by orbital period, the sample reveals a tendency for the short period systems to have cooler white dwarfs. This could be due to a combination of two evolutionary effects, namely age and accretion rate. When the WD temperatures are looked at as a function of the the orbital inclination, there is no obvious correlation for intermediate values; whereas the highest inclination systems inexplicably tend to have the lowest temperatures and the shortest orbital periods. The absence of data for inclinations below about $20^{\circ}$ probably reflects the difficulty of determining system parameters in these cases. Thus it is important to investigate any possible selection effects.

For a good fraction of the systems we cannot fit a DA WD and the spectrum does not resemble an optically thick accretion disc either. But at least much of the short wavelength spectrum, if not all the IUE range, can be fitted well with a DB WD, despite the fact that there is no theoretical explanation for this, given that the WD is accreting hydrogen-rich material from the secondary star. Clearly, this aspect requires further investigation.

\section{Conclusions}

- The IUE archive provides a unique opportunity to perform statistical studies of the white dwarfs in quiescent dwarf novae.

- The temperatures obtained give reasonable agreement with detailed models using recent HST data of a few systems so far observed.

- In about half of the systems, the best (or even only) fit is obtained with a DB WD.

- There is a correlation of $T_{\mathrm{WD}}$ with orbital period.

- Most of the coolest white dwarfs appear in the short period, high inclination systems.

\section{References}

Horne, K., Marsh, T.R., Cheng, F.H., et al., 1994, Ap. J., 426, 294

la Dous, C., 1996, in preparation. 